\title{
Konzeptualisierung der Emotionen auf der Mikrotextebene (am Beispiel von Sprichwörtern mit der Komponente Zorn)
}

\author{
Anna Gondek (Breslau)
}

\begin{abstract}
In this paper I would like to present the emotionality and emotions in German proverbs on the example of proverbs with the component anger. I am focused not on theoretical considerations about the proverb but on the analysis of the collected language material. The component anger is one of the highly productive proverbial components, it has been found in over four hundred units. It should be noted that most of the proverbs feature the negative effects of this emotion. The analysis of the material allowed for the extraction of nine main groups which show how anger is perceived and described in German proverbs. For example: anger blinds people, makes them lose self-control and brings about serious consequences. Although currently proverbs are often negatively labelled as simple wisdom of ordinary people, I think that they have not entirely lost their social and educational content.
\end{abstract}

\section{$1 \quad$ Sprichwort}

In dem vorliegenden Beitrag möchte ich am Beispiel ausgewählter Sprichwörter einen Einblick verschaffen, wie die Emotionalität und Emotionen in deutschen Sprichwörtern zu Wort gebracht werden. Es wird geplant, für das deutsche Korpus sein polnisches Pendant zusammenzustellen, um auf diese Weise ein konfrontatives Bild zu erbringen. An dieser Stelle sei darauf hingewiesen, dass es nicht meine Absicht ist, das Sprichwort theoretisch zu behandeln, Definitorisches zu erörtern oder auf die einzelnen Aspekte der Sprichwortforschung einzugehen. Im Bereich meines Interesses liegt hauptsächlich die Frage, wie in den deutschen Sprichwörtern über Emotionen gesprochen wird und welche Meinungen der deutschen Sprachgemeinschaft über die gewählte Emotion den Eingang in die Parömien gefunden haben. Deswegen werden hier nur kurz und stichwortartig die wichtigsten Merkmale der Sprichwörter genannt.

Die meisten Definitionen des Sprichwortes heben hervor, dass es "in sich geschlossene, festgeprägte Sätze" sind (vgl. Seiler 1922: 2; Röhrich/Mieder 1977: 2; Fleischer 1997: 76; Burger 2010: 106), die "allgemeine Aussagen oder Urteile" (Burger 1973: 54) enthalten. "In den Sprichwörtern sind Erfahrungen der einfachen Menschen verallgemeinert" (Fleischer 1997: 77), sie drücken "eine Lebensregel oder Weisheit in prägnanter, kurzer Form" aus (Röhrich/Mieder 1977: 3). Sprichwörter seien "didaktisch [...], weil sie Erfahrungen und Weisheit lehren, Moral predigen, weil sie als 'Wahrwörter' Autorität beanspruchen" (Röhrich 1994: 18). Auf die "lehrhafte Tendenz" (Seiler 1922: 2), "Lehrhaftigkeit" (Röhrich/Mieder 1977: 2), 
"Verbürgung von Wahrheit" oder "Belehrung" (Burger 2010: 112-118) wird oft in den Erwägungen über das Sprichwort hingewiesen. Hervorgehoben wird auch ihre allgemeine Bekanntheit - "Volksläufigkeit" (Seiler 1922: 2), "Geläufigkeit" und "kulturell-soziale Gebundenheit" (Komenda-Erle 2007: 416). Neben dem "generalisierend-referentiellen Aspekt" (Burger 2010: 107) wird darauf hingewiesen, dass Sprichwörter zum Handeln Ansporn geben können, indem sie "zugleich Anweisungen für das Handeln oder Deutungen des Handelns in den von ihnen modellierten Situationen" sind (ebd. 2010: 107). Ähnliche Meinung vertritt Röhrich, der behauptet, dass ein Sprichwort "eine unser Verhalten betreffende Einsicht oder eine Aufforderung zu einem bestimmten Verhalten ausspricht" (1994: 18). Nach Burger (2010: 107-108) hat dieser "Handlungsaspekt mindestens zwei Dimensionen", es sind die "soziale Funktion" und die "kontextuellen Funktionen". Es handelt sich nämlich darum, dass Sprichwörter einerseits Meinungen, Lebensregeln, Normen formulieren, andererseits gewisse Aufgaben in den Kommunikationssituationen übernehmen können.

\section{Sprichwort als Text}

Für die Betrachtung des Sprichwortes als ein (Mini-)Text plädierte Fleischer, der in seinen Beiträgen, in denen er die Ähnlichkeiten und Unterschiede zwischen Phraseologismen und Sprichwörtern herauszuarbeiten versuchte, die Meinung vertritt, dass die Phraseologismen lexikalische Einheiten und Sprichwörter dagegen Texte sind. Das einzelne Sprichwort wurde von ihm als "Text und Repräsentant einer spezifischen Textsorte, ein Element der literarischen Tradition" (Fleischer 1994: 155) aufgefasst. Ein wichtiges Unterscheidungsmerkmal ist nach Fleischer die Tatsache, dass Sprichwörter "aus dem Gedächtnis als Texte zitiert" (ebd.: 156), Phraseologismen dagegen reproduziert werden. "Das Sprichwort zitieren heißt: Der Sprecher gibt ausdrücklich zu verstehen, dass der betreffende Satz, der eine Aussage mit Verallgemeinerungsanspruch fixiert, nicht vom Sprecher selbst stammt, [...], sondern, dass er ihn nur "wiedergibt", sich berufend auf eine höhere Instanz" (ebd.: 156). Fleischer deutet darauf hin, dass Sprichwörter, so wie Rätsel, Volkslied, Märchen oder Volkswitz in der literarischen Tradition der Volksdichtung stehen und als repräsentative Einzeltexte (oft mit Varianten) überliefert werden (vgl. ebd: 157).

Auch in der parömiologischen Tradition hat man das Sprichwort als einen zur Volksdichtung gehörenden Text erforscht, "mit dem Schwerpunkt weniger auf linguistischem als vielmehr auf volkskundlichem und kulturgeschichtlichem Gebiet" (Fleischer 1994: 158). Zwar wurden hier die Merkmale angeführt, die die These unterstützen, dass das Sprichwort als eine Textsorte betrachtet werden kann, es fällt aber zugleich auf, dass es eine sehr spezifische Struktur hat, es ist nämlich "ein Text in Satzstruktur, und zwar in Ein-Satz-Struktur" (Fleischer 1994: 159). Einbezogen werden Satzgefüge bzw. Satzverbindungen sowie die Auslassungssätze (ebd.: 159). Die Ansicht bestätigt Burger, indem er meint, dass Sprichwörter "als selbständige 'Mikrotexte' aufgefasst werden können [...] und keine textlinguistische Anpassung an einen Kontext [benötigen]" (Burger 2010: 106).

Die Einbettung der Sprichwörter in die Äußerungen ist spezifisch, sie werden als ganzheitliche Elemente angeführt, die innerhalb einer Äußerung oder einer größeren Texteinheit relativ autonom erscheinen und nicht so eng wie Phraseologismen mit dem Text verbunden sind. Oft werden sie durch metasprachliche Elemente angekündigt, wie etwa: "Man könnte zu Beginn zwei 
(www.edumagazin.de/313000/Man_koennte_zu_Beginn_zwei_Sprichwoerter_zitieren_und_z war_einmal_Was.html, 05.07.2015), "Ein arabisches Sprichwort lautet: [...]" (www.codecheck.info/suesswaren_snacks/frucht_weingummi/ean_7640104959014/id_81994 9/Biolini_Salbei_Bonbons.pro, 05.07.2015) oder "Ich muss in diesem Falle eines der von mir am meisten gehassten Sprichwörter zitieren: [...]" (www.1000fragen.de/projekt/fragen /frage.php?qid=2072, 05.07.2015).

Die Sprichwörter können meistens ohne den umgebenden Kontext verstanden werden (vgl. Burger 2010: 106). Es sind kurze und prägnante Formulierungen, die bildlich oder nicht, sich von den gewöhnlichen "generischen Aussagen" (Fleischer 1991: 7) unterscheiden durch "die geprägte Sprichwortform, mit der der Autoritätsanspruch des Zitats verbunden ist" (ebd. 1991: 7). Diese Bezogenheit auf eine höhere Instanz oder Autorität soll daraus resultieren, dass in Sprichwörtern Erfahrungen und Erkenntnisse der jeweiligen Sprachgemeinschaft zusammengefasst und tradiert werden. Typische, stets wiederkehrende Situationen, Verhaltensweisen, Handlungen der Menschen werden in ihnen verallgemeinert, in einfache Regeln gefasst, oft erklärt und kommentiert. Daher ist im Sprichwort nicht nur die propositionale, sondern auch die illokutive Komponente so wichtig, z. B. Bewertung, Verhaltensanweisung, Legitimitätsnachweis (vgl. Fleischer 1991: 7), Ratschlag, Belehrung, Aufforderung, Warnung usw. Das gleiche Sprichwort kann dabei, abhängig von der Kommunikationssituation, unterschiedliche illokutive Funktionen erfüllen. Man kann des Weiteren vermuten, dass durch die Verwendung von Sprichwörtern eine Wirkung beim Adressaten, Gesprächspartner hervorgerufen wird (eventuell hervorgerufen werden soll), also die Äußerung bewusst oder unbewusst eine Wirkung erzielt, demnach perlokutive Akte vollzogen werden können.

Die von mir analysierten Sprichwörter stammen aus dem Deutschen Sprichwörterlexikon von Karl Friedrich Wilhelm Wander, also aus einem Werk, das am Ende des 19. Jahrhunderts entstanden ist. Ich habe mich für dieses Werk bewusst entschieden, da es bei der geplanten Analyse nicht die linguistische Untersuchung der Sprachkorpora meinem Interesse galt, sondern vor allem ein durch Sprichwörter von Generation zu Generation tradiertes und in ihnen verfestigtes Bild von Emotionen. Von Bedeutung ist hier die Art und Weise, wie in den Formulierungen, die ihren festen Platz im Sprachbestand haben, die Emotionen konzeptualisiert werden. Diese Vorgehensweise möchte ich damit legitimieren, dass es seit dem 20. Jahrhundert zu einer großen Umwandlung in der Verwendung von Sprichwörtern gekommen ist. Die Sprichwörter sind zwar aus dem alltäglichen sprachlichen Gebrauch nicht völlig verschwunden, ihre traditionelle Form und Verwendungsweise unterliegen jedoch starken Veränderungen, was die Untersuchungen bestätigen (u. a. Mieder 2004; Burger 2010: 120-124). In den Vordergrund treten die sprachspielerischen Modifikationen, Verfremdung und die beabsichtigte Abkehr von der traditionellen, seit Generationen wiederholten "Volksweisheit". Dies alles verursacht, dass Sprichwörter und ihre Wahrheiten oft nicht mehr "ernst" genommen werden und dadurch die Rolle, die ihnen in der gesellschaftlichen Kommunikation bisher zukam, eingebüßt haben. In meinem Beitrag beabsichtige ich dagegen, die seit Generationen tradierten Meinungen zu erfassen, die alten Erfahrungsätze darzustellen. Die Resultate der so konzipierten Analyse kann man ferner mit Ergebnissen einer korpusbasierten Untersuchung konfrontieren, um u. a. festzustellen, ob die bei Wander genannten Sprichwörter bis jetzt verwendet werden, wie ihre Frequenz ist, ob sie ihre Form behalten haben (z. B. modifiziert oder 
nicht modifiziert auftreten). Es eröffnet jedoch weiterführende Dimensionen, die den geplanten Rahmen dieses Beitrags sprengen würden.

\section{Emotionen in Sprichwörtern}

Die Emotionen, sowohl positive als auch negative, haben einen sehr großen Einfluss auf das menschliche Leben. Da die meisten Menschen in einer Gemeinschaft leben, beeinflussen die Emotionen eines Individuums zugleich seine Nächsten, Bekannten und diejenigen, die mit jemandes Emotionsausbrüchen konfrontiert werden. Es ist also ein Lebensbereich, das oft durch festgeprägte/feste Konstruktionen verbalisiert wird (es können z. B. zahlreiche Phraseologismen gefunden werden, die Emotionen thematisieren und zum Ausdruck bringen). Dieses Thema kann doch unmöglich bei Sprichwörtern unbeachtet bleiben. Es sind unter den Sprichwörtern solche zu finden, die in ihrem Bestand Komponenten enthalten, die einzelne Emotionen bezeichnen. Eine hohe Produktivität weisen, z. B. die Komponenten: Angst (46), Scham (150), Furcht (201) oder Freude (239) auf.

Dabei lassen sich zwei sprachliche Verfahren beobachten - ein Sprichwort ist eine allgemeine bildhafte, metaphorische oder direkte Aussage über Emotionen:

1. Ohne dass darin Komponente oder Komponenten, die Emotion(en) bezeichnen, genannt werden, jedoch bezieht sich die gesamte Aussage auf Emotionen, z. B.:

- Auch Ameisen haben Galle. (auch der Schwächste und Gutmütigste kann zuletzt zornig werden) ${ }^{4}$;

- Wenn der Donner brüllt, schüttert die Erde;

- Wer den Schuh tregt, der weiss seinen tuck, wo er ihn am ergesten druck. ${ }^{5}$ (Heimlichen Kummer kennt nur der, den er drückt. Nicht allemal ist der glücklich, der so aussieht) ${ }^{6}$;

- Kommt ein alter Gaul in Gang, so ist er nicht zu halten. (So weiss auch ein alter Geck, wenn er einmal lustig ist, seiner Laune keine Grenze zu setzen)7;

- Besser singen als fluchen.

2. Mit der Komponente bzw. Komponenten, die Emotion(en) bezeichnen, und die gesamte Aussage bezieht sich auf Emotionen, z. B.:

- Fleissig vnd kunstreich sein ohne Danck, macht verdross, zornig vnd kranck;

- Ein zornig Man ist seiner Sinn beraubt;

- Auf Weh und Ach folgt Freude nach;

- Betrübniss hangt immer an der Freude;

- Heute Freude, morgen Leid;

\footnotetext{
${ }^{4}$ Die in Klammern angeführten Kommentare stammen von Wander (www.zeno.org/Wander1867/A/Ameise?hl=ameisen, Nr. 3 [05.07.2015]).

${ }^{5}$ In Beispielen wurde die alte Schreibweise Wanders beibehalten.

${ }^{6}$ Vgl.: www.zeno.org/Wander-1867/A/Schuh?hl=ergesten, Nr. 67 [05.07.2015].

${ }^{7}$ Vgl.: www.zeno.org/Wander-1867/A/Gaul?hl=alter+gaul, Nr. 42 [05.07.2015].
} 
- Frauen lachen, wenn sie können, und weinen, wenn sie wollen;

- Sehr fröhlich ist gefährlich, sehr traurig ist beschwerlich;

- Die am Abend lustig singen, am Morgen oft die Hände ringen;

- Ein fröhlicher Wirth, macht fröhliche Gäst.

Die angeführten Beispiele zeigen, dass in der ersten Gruppe bildhafte Sprichwörter überwiegen, die erst gedeutet werden sollen. Die verwendeten Bilder sind aber gut motiviert und der Inhalt ist eher leicht verständlich. Die Anzahl der indirekten Sprichwörter ist wesentlich geringer als deren, die die Emotionen direkt nennen.

Die zweite Gruppe umfasst vor allem direkte Aussagen und Feststellungen. Die Emotionen werden beim Namen genannt, es werden verschiedene Aspekte der Emotionen bzw. der Emotionalität angesprochen. Es lassen sich gewisse illokutive Funktionen der zitierten Sprichwörter erkennen, z. B. Warnung (Auch Ameisen haben Galle) oder Trost (Auf Weh und Ach folgt Freude nach), Hinweis, aber zugleich ein situationsgebundenes Kommentar (Ein fröhlicher Wirth, macht fröhliche Gäst). In dem Fall ist die Anzahl der Sprichwörter weit höher, an den Beispielen ist zu sehen, dass manche Sprichwörter gleich über zwei unterschiedliche emotionale Zustände sprechen, die miteinander kontrastiert werden, z. B.: Weh und Betrübnis mit Freude, lachen mit weinen, fröhlich mit traurig. Bemerkenswert ist das Sprichwort: Die am Abend lustig singen, am Morgen oft die Hände ringen, wo einer der emotionellen Zustände direkt, mit dem Wort lustig genannt wird, die kontrastierende Gemütslage dagegen aus der Phrase Hände ringen erstellt werden muss, nämlich einer Trauergeste oder Gebärde der Verzweiflung.

Wegen der großen Anzahl der im Wörterbuch angeführten Sprichwörter habe ich mich entschieden, eine repräsentative Auswahl von Parömien mit der Komponente Zorn zum Gegenstand meiner Analyse zu machen.

\section{Sprichwörter mit der Komponente Zorn}

Der Zorn ist nach Duden (2001:1862) ein: "heftiger, leidenschaftlicher Unwille über etwas, was jemand als Unrecht empfindet oder was seinen Wünschen zuwiderläuft". Bei Büntig (1996: 1346) wird Zorn als "wütendes, heftiges, oft lautstark geäußertes Gefühl, das sich gegen eine Person od. Sache richtet" definiert.

Es konnten bei Wander unter dem Stichwort Zorn 206 sprichwörtliche Einheiten festgestellt werden. Man kann außerdem auf andere Fundstellen hinweisen, wo die Sprichwörter mit der Komponente Zorn auftreten, z. B.: Afterred' und Zorn gehören nicht über Tisch oder Bauern und Arme haben auch ihren Zorn, er hat aber die Wehre nicht, Eine Antwort sanft und lind, stillet den Zorn geschwind. Die Anzahl der Sprichwörter, die den Zorn auf verschiedene Weise darstellen und kommentieren ist also noch wesentlich höher - insgesamt konnten zusätzliche 210 Belege ermittelt werden. Eine ergänzende Gruppe bilden Sprichwörter mit den Komponenten zornig und Zorniger, z. B.:

- Ein zornig Mann das recht nicht sehen kann;

- Ein zornig Mann hat seine Sinn biss ohn funff; 
- Ein zornig Mann ist nicht bey ihm selbst;

- Ein zornig Mann richt Hader an;

- Ein zorniger Mensch hat den Teufel im Leibe;

- Der Zornig hört nicht auf vernünftig Wort;

- Wems vbel geht, bald zornig wirt, ob jhn gleich nur ein strohalm jrrt;

- Narren werden bald zornig (entrüstet);

- Schafe werden auch zornig;

- Ein Schmeichler darf nicht zornig werden (Sonst fällt er aus der Rolle);

- Geschwind zornig, geschwind versöhnt;

- Was man zornig thut, ist selten recht vnd gut;

- Zornig sein bringt Noth und Pein;

- Zornig sein heisst halb wahnsinnig sein;

- Zornig und hitzig ist niemals witzig.

Die von obigen Sprichwörtern verbalisierten Meinungen decken sich größtenteils mit denjenigen, die die Komponente Zorn beinhalten.

Im Folgenden möchte ich mich auf die meist angesprochenen Bereiche konzentrieren, die in Sprichwörtern mit der Komponente Zorn thematisiert werden.

1. Der zornige Mensch ist unfähig zur Einsicht, zur richtigen Einschätzung der Lage:

- Der Zorn macht blind;

- Der Zorn wirft blinde Junge, wie die Hündin;

- Des Zornes Anfang ist rasende Blindheit und das Ende reuige Blödigkeit;

- Zorn, Buhlschaft, Wein und Spiel verblenden viel;

- Zorn den Menschen so verblend, dass er sich selbst nicht kennt.

2. Der Zorn macht die Menschen töricht - nur Toren werden zornig:

- Beim Zorn kennt man den Thor'n;

- Im Zorn erkennt man den Thor'n;

- Der Zorn erwürgt den Thorn;

- $\quad$ Der Zorn ist die Blüte der Narrheit;

- Der Zorn ist ein Narr;

- Zorn wohnt in des Narren Hertzen;

- Wenn der Zorn zu Rathe sitzt, öffnet eine Thorheit der andern die Thür;

- Des Mannes weissheit ein ende hat, wenn grosser zorn jhm begaht;

- Geduld ist die Schwester der Weisheit, Zorn der Bruder der Narrheit. 
3. Der Zorn verursacht den Verlust der Selbstkontrolle und der Selbsthemmung:

- Wer im Zorn handelt, weiss selten, was er thut;

- Der Zorn weiss nicht, was er thut;

- Im Zorn weiss man nicht, was man thut.

4. Der Zorn bringt unterschiedliche schlimme Folgen mit sich:

- Der Zorn erzeugt keine guten Kinder;

- Im Zorn wird nichts Gutes gestiftet;

- Der Toll Zorn thut mehr schaden als drey Dreschflegel;

- Der Zorn kann in einem Augenblick mehr verderben, als der Verstand in einem Tage gut machen kann;

- Der Zorn bringt greuliche Gäste mit sich;

- Jäher Zorn stiftet viel Böses;

- Der Zorn entstellet das Gesicht und hält kein recht Gericht;

- Der Zorn gebiert Hass;

- Zorn gebiert Zorn;

- Im Zorn geht Freundschaft verlor'n;

- Zorn macht aus Freunden Feinde;

- Im Zorn hat offt ein Mensch gethan, was ihm sein Lebtag hanget an;

- Der Zorn thut jedem Verbrechen die Thür auf.

5. Es ist nicht gut im Zorn Entscheidungen zu treffen:

- Der Zorn ist ein schlechter Rathgeber;

- Zorn hat nie Rath gebracht;

- Der Zorn geht zur That ohne Weisheit und Rath;

- Wo Zorn ist, ist weder Weissheit noch Rhat;

- Was man im Zorn thut, ist selten gut;

- Es ist selten gut, was einer aus zorn thut.

6. Der Zorn löst die Zunge auf, durch Zorn wird der menschliche Verstand beeinträchtigt:

- Im Zorn spricht mancher Mann das ergest, was er (denken) kann;

- Im Zorn redet und thut man nichts Gescheites;

- Zorn kann nicht immer die Zunge im Zügel halten;

- Der Zorn kann die Zunge nicht bezähmen;

- Zorn bringt an den Tag, was im Herzen verborgen lag; 
- Der Zorn ist ein kurzer Wahnsinn;

- Zorn ist ein kurz Unsinnigkeit;

- Wo der Zorn einkehrt, muss der Verstand ausziehen;

- Im Zorn ist die Rach Meister und nicht der Verstand.

7. Der Zorn schadet dem Zornigen, macht sein Leben kürzer; der Zornige tut sich selbst einen Schaden an:

- Der Zorn lässt den Menschen nicht alt werden;

- Der Zorn verkürzt das Leben;

- Zorn schneidet das Leben ab;

- Der Zorn wird nicht alt;

- Der Zorn wird sein eigner Henker;

- Zorn ist alten Mannes Tod;

- Des Zornes Feuer ergreift zuerst seinen Herrn;

- Verhaltener Zorn ist für Leib und Seel' ein Dorn;

- Wer im Zorn handelt, geht im Sturm unter Segel;

- Zorn schadet niemand mehr, als dem Zornigen;

- Zorn sticht andere mit Nadeln und sich selbst mit Dolchen;

- Lass gähen Zorn nit in dein Hertz, dann er bringt nur Schmerz.

8. Nach dem heftigen Zornausbruch kommen Besinnung und Reue:

- Auf grossen Zorn folgt grosse Reu;

- Dem Zorn gehet die Rew auff den Socken nach;

- Des Zornes Anfang ist rasende Blindheit und das Ende reuige Blödigkeit;

- Der Zorn fängt mit Unsinnigkeit an und endigt mit Reu und Scham;

- Des Zorns Ausgang (Ende) ist der Rew Anfang;

- Geht der Zorn, so kommt die Reue.

9. Man soll sich dem Zorn nicht ergeben und ihn in Schranken halten; es ist lobenswert, seinen Zorn bezwingen zu können:

- Der Zorn soll nicht Obrister, sondern Trabant sein;

- Den Zorn bey dir lass nicht regiern, dan das Gemüth thut er verfürn;

- $\quad$ Man muss dem Zorn nicht die Zügel schiessen lassen;

- Man soll dem Zorn nicht Raum geben;

- So lang der Zorn hat überhand, kannst du nicht sein ein Mensch genannt;

- Von Zorn hält der Verständige sich frei; 
- Der Zorn beherrscht nur schwache Leute;

- Wer den Zorn erlegt, der erlegt seinen grössten Feind;

- $\quad$ Wer den Zorn überwindet, besiegt einen grossen Feind;

- $\quad$ Wer im Zorn sich mässigen kann, ist ein starker (weiser) Mann;

- Wer seinen Zorn beherrschen kann, ist ein edler (starker) Mann;

- Wer seinen Zorn bezwingen kann, das ist ein tapferer Mann;

- Wer seinen Zorn bezwingt, hat einen (grossen) Feind besiegt;

- Der ist ein tapferer Mann, der seinen Zorn bemeistern kann;

- $\quad$ Der ist fürwahr ein weiser Mann, der seinen Zorn bezemen kan;

- Bringt dein Gesinde dich in Zorn, thu, dass du bleibest unverworr'n.

Die oben erwähnten Sprichwörter nennen auf eine beinahe synonyme Weise die negativen und gar schlimmen Folgen der Zornausbrüche. Ein weiser Mensch soll sich dem Zorn nicht ergeben und weiß seinen Zorn zu zähmen, sonst kann er schwer unter dessen Folgen leiden. Im Punkt 3 wird zusätzlich auf physiologische Folgen des Zornausbruches hingewiesen, das Gesicht des Zornigen ist entstellt, verändert.

Unter den Sprichwörtern mit der Komponente Zorn sind überdies solche zu finden, in denen behauptet wird, dass diese Emotion etwas Positives mit sich bringt, z.B. stärkend wirken kann:

- Zorn macht stark;

- Zorn und Schmerz bringen Muth und Stärke;

- Stärke wird durch Zorn gewetzt;

und in manchen Fällen gerechtfertigt und nötig ist, wo man von zwei Übeln das kleinere wählen muss:

- Besser ein kleiner Zorn als ein grosser Schade;

- Besser kleiner Zorn, als grosse Schande.

Der Zorn kann sogar als Prüfstein menschlichen Verhaltens dienen:

- Am Geld, im Trunk und im Zorn erkennt man des Menschen Gemüth;

- Drei Dinge können nur an drei Orten erkannt werden: die Tapferkeit im Kriege, die Weisheit im Zorn und die Freundschaft in der Noth.

Es wird darauf hingewiesen, dass der Zorn in gewissen Situationen eine legitime und nötige Reaktion ist:

- Wer nicht um Noth Zorn hat, das en ist nicht eines weisen Rath;

- Wer kein Zorn hat, der hat kein Verstand.

Insbesondere wird die positive, stärkende Auswirkung des (kleinen) Zornes auf die Liebe hervorgehoben: 
- Ein kleiner Zorn stärkt die Liebe;

- Der Buhler Zorn ist der Liebe (oder der: Buhlschaft) Sporn.

Zornig sein ist laut Sprichwort männlich:

- Der ist kein Mann, den Zorn nicht warm machen kann;

- Zorn ist ein Mann, Sanfftmuth eine Fraw.

Das letztzitierte Sprichwort weist auf die traditionelle Rolle der Frau, die den männlichen Zorn stillen und besänftigen soll:

- Ein fromme Fraw senfftet des Mannes zorn;

- Eine anmuthige Frau sieht ihrem Manne den Zorn aus dem Gesicht.

Wie bekannt, können die Sprichwörter entgegengesetzte Meinungen vertreten, was in diesem Korpus bestätigt werden konnte, nämlich:

- Sich den Zorn lassen übergehn, ist weibisch;

- $\quad$ Es ist kein Zorn über Weiber Zorn.

In Sprichwörtern wird überdies der Versuch unternommen, die Fragen zu beantworten:

a. wodurch der Zorn hervorgerufen wird:

- $\quad$ Alte Geschichten zu erzählen, macht neuen Zorn;

- Viel sprechen erregt Zorn;

- Ein Wort kann viel Zorn anrichten;

- Vier ding gebehren den zorn: verlachung, schmähung, vndankbarkait; gerechtigkait, warhait;

- Das Gesetz richtet nur Zorn an;

- Das Gesetz ist Zorn, das Evangelium genad;

b. auf welche Weise der Zorn zu stillen ist:

- Mit Geduld und Schweigen stillet man viel Zorn;

- Stillschweigen vnd Gedult stillt viel zorn;

- Ein heimliche gab stillet den zorn;

- Geschenke stillen den Zorn;

- Bellenden Hunden gibt man Brodt, so hat mann vor jhrem Zorn keine Noth;

- Nachgeben stilt viel Zorn;

- Nachlassen stillt vil zorn;

- $\quad$ Sanfte Rede stillt (besänftigt, mässigt) den Zorn;

- $\quad$ Sanfte Worte stillen den Zorn;

- $\quad$ Sanftmuth stillt den Zorn; 
- Wohlthat stillt den Zorn;

- Ein gut Wort stillet grossen Zorn;

- Zeit stillet den Zorn;

- Zeit und Weil' stillt Zorn in Eil'.

Es fällt auf, dass ausgesprochen hochgeschätzte Werte, wie Gerechtigkeit und Wahrheit, paradoxal den Zorn erwecken können. Die Anzahl der Sprichwörter unter b. kann davon zeugen, dass man mit verschiedenen Mitteln versuchte, jemandes Zorn zu stillen und dass es seit eh und jäh eines guten Rats bedurfte. Es wurden eher mildernde Mittel empfohlen, wie Ausdauer im ruhigen Ertragen, Geschenke (Gunst-Erkaufen), Nachgeben, Friedfertigkeit oder Abwarten. Nicht empfohlen dagegen war eine scharfe verbale Reaktion:

- Zorn wird mit Scheltworten nicht gestillt.

Man soll den Zorn nicht hegen und die Versöhnung anstreben:

- $\quad$ Ueber dem Zorn soll die Sonne nicht untergehen;

- Man soll die Sonne nicht lassen über seinem Zorn untergehen.

Viele Sprichwörter heben hervor, dass ein ohnmächtiger Zorn und Zorn kleiner, armer Leute lächerlich ist:

- Der Zorn des Schwachen ist zum Verlachen;

- Zorn ohne Macht wird verlacht (ausgelacht);

- Ohne Macht ist der Zorn eitel;

- Kleiner Herren Zorn macht nicht viel verworr'n;

- Armer Leute Zorn macht nicht viel verworr'n;

- Geringer Leute Zorn ist nicht werth ein Haferkorn;

- Eines armen Mannes Zorn wird verlacht.

Der Zorn der Starken und Mächtigen ist dagegen zu befürchten:

- Wenn ausbricht grosser Herren Zorn, sind arme Leute gar verlor'n;

- Der Herren Zorn ist schwer.

Man muss aber damit rechnen, dass selbst die unwichtigsten und gutmutigsten Leute in Zorn geraten können:

- Kleine Leute haben auch Zorn;

- Die Schaff haben auch Zorn;

- Auch eine Taube hat Zorn.

Thematisiert in Sprichwörtern wird die bekannte Tatsache, dass diejenigen, die einst eng befreundet waren oder einander liebten, oft im Hass und Zorn auseinandergehen. Intensive positive Gefühle verwandeln sich in stark negative Emotionen:

- Je näher freundt, je bitterer zorn; 
- Die sich aus Liebe nehmen, laufen oft aus Zorn auseinander;

- Grosse lieb gebieret grossen zorn.

\section{Sprichwörter mit der Komponente Zorn samt anderen Komponenten}

Neben den Sprichwörtern, die die Komponente Zorn enthalten, gibt es eine Reihe von solchen, die zusätzlich andere Komponenten beinhalten.

Es sind Emotionen, Affekte, Gefühle und menschliche Laster. Ihre Verflechtung mit dem Zorn zieht bedauerliche Folgen nach sich, z. B.:

- Liebe und Zorn machen alle Ding' verworr'n;

- Zorn und Liebe geben schlechten Rath;

- Zorn und Lust sind böse Rathgeber;

- Auss Zorn und Begierd gar nichts gutes wird;

- Begierde und Zorn sind böse Rathgeber;

- Eifferen vnd zorn verkürtzen das leben;

- Uebermuth und Zorn machen all Ding verworr'n;

- Neid und Zorn würgt den Thorn;

- Hass vnd Zorn alten langsam;

- Zorn und Wuth nicht lange leben thut;

- Geitz, zorn, böss lieb, drei laster sind inn schand vnd spot sie stürtzen geschwind.

Der Zorn gepaart mit einer tadelnswerten Verhaltensweise hat genauso schlimme Konsequenzen:

- Buhlschaft, Spiel und Zorn verblenden Weise und Thor'n;

- Gewalt vnd zorn beysammen ist der todt;

- Gewalt vnd zorn machen alle ding verworr'n.

Ebenfalls bilden Geld und Zorn eine gefährliche Mischung:

- Geld vnd Zorn macht alle Dinge verlohrn;

- Gibst kein Geld, so kommt der Zorn, gibst das Geld, ist Freud' verlor'n.

Eine charakteristische Gruppe besteht aus Sprichwörtern in denen drei Dinge genannt werden, wie z. B.:

- Drei Dinge machen aus den Weisen Thor'n: Argwohn, Hoffart, Zorn;

- Drei Dinge seindt schwer: die Steine, der Sand, der narren Zorn;

- Drei Dinge soll man nicht zu sehr reizen: die Brüste, die Nase, den Zorn.

Die oben präsentierten Beispiele umfassen die Mehrheit der ermittelten Einheiten. Es wurden mehrere Varianten angeführt, um die darin enthaltenen Konzepte und Vorstellungen mehrdimensional erscheinen zu lassen. Aus Platzgründen konnten nicht diese Bereiche thematisiert 
werden, denen vereinzelte Sprichwörter zugrunde liegen. In dem Fall wäre eine separate Studie nötig.

\section{$5 \quad$ Fazit}

Die Komponente Zorn gehört zu den hochproduktiven sprichwörtlichen Komponenten, es konnten über vierhundert Einheiten ermittelt werden. Es ist festzustellen, dass die meisten Sprichwörter die negativen Folgen dieser Emotion nennen. Der Zorn verblendet die Menschen, verursacht, dass sie ihre Selbstkontrolle und ihre Selbsthemmung in ihren Äußerungen und Reaktionen verlieren und zu unvernünftigen, gar wahnsinnigen Handlungen fähig sind. Die Entscheidungen, die unter dem Einfluss dieses Affekts getroffen werden, sind selten gut. Daher haben Zornausbrüche für den Zornigen und seine Umgebung verschiedene schlimme Folgen. Sie können sogar seine Gesundheit beeinträchtigen, geradezu sein Leben kürzen. Lob und Anerkennung verdienen diejenigen, die ihren Zorn zu zähmen vermögen. Zorn entwickelt sich nicht geschlechtsspezifisch, sowohl Frauen als auch Männer können darin geraten. Es wird jedoch von einer (sanftmütigen, duldsamen) Frau erwartet, den männlichen Zorn zu beschwichtigen. In Sprichwörtern gibt es überdies verschiedene Ratschläge, womit und auf welche Weise man jemanden in seinem Zorn beruhigen kann. Mit einer gewissen Resignation stellt der Volksmund fest, dass der Zorn der kleinen Menschen nur zum Verlachen ist, der Mächtigen dagegen gefährlich sein kann. Wenn sich mit dem Zorn noch andere negative Emotionen, Laster und allgemein nicht akzeptierte Verhaltensweisen verbinden, zieht das gewiss schlimme Konsequenzen nach sich. Die einzige positive Wirkung der sonst negativ empfundenen Emotion ist, dass man durch Zorn an Stärke gewinnen kann.

Die Sprichwörter werden in unserem Zeitalter oft mit dem geringschätzenden Etikett einfache Weisheiten kleiner Leute abgetan, die längst überholt sind und nicht mehr zu unserer modernen Lebensauffassung passen. Die Tatsache jedoch, dass sie ein Resultat jahrhundertelanger menschlicher Beobachtungen und Erfahrungen sind, verursacht meiner Meinung nach, dass sie ihren sozialerzieherischen Inhalt nicht ganz verloren haben. Deshalb sollen wir sie nicht abwertend betrachten, denn die menschliche Natur scheint in der zwar neuen Umgebung unveränderlich zu bleiben.

\section{Literatur}

Bünting, Karl-Dieter (1996): Deutsches Wörterbuch. Chur: Isis.

Burger, Harald (2010): Phraseologie. Eine Einführung am Beispiel des Deutschen. 4. neu bearbeitete Auflage. Berlin: Erich Schmidt. (= Grundlagen der Germanistik 36).

Burger, Harald/Jaksche, Harald (1973): Idiomatik des Deutschen. Tübingen: Niemeyer.

Das 1000Fragen-Projekt. www.1000fragen.de/projekt/fragen/frage.php?qid=2072 [25.04.13].

Duden (2001): Deutsches Universalwörterbuch 4. neu bearbeitete und erweiterte Auflage Dudenredaktion (ed.). Mannheim: Duden.

EduMagazin. www.edumagazin.de/313000/Man_koennte_zu_Beginn_zwei_Sprich-woerter_ zitieren_und_zwar_einmal_Was.html [25.04.13].

Fleicher, Wolfgang (1997): Phraseologie der deutschen Gegenwartssprache. 2. durchgesehene und ergänzte Auflage. Tübingen: Niemeyer.

Fleischer, Wolfgang (1991): "Zum Verhältnis von Parömiologie und Phraseologie". Niederdeutsches Wort. Beiträge zur niederdeutschen Philologie 31: 3-13. 
Fleischer, Wolfgang (1994): "Phraseologismus und Sprichwort: lexikalische Einheit und Text". In: Sandig, Barbara (ed.) (1994): Europhras 92. Tendenzen der Phraseologieforschung. Bochum, Brockmeyer: 155-172.

Komenda-Erle, Barbara (2007): "Zu den lexikographischen Aspekten von Sprichwörtern anhand von einsprachigen deutschen Wörterbüchern". In: Bartoszewicz, Iwona/Szczęk, Joanna/Tworek, Artur (eds.) (2007): Fundamenta linguisticae. Wrocław/Dresden, ATUT/Neisse: 415-426. (= Linguistische Treffen in Wrocław 1).

Mieder, Wolfgang (2004): "Antisprichwörter und kein Ende. Von sprachlichen Eintagsfliegen zu neuen Sprichwörtern". In: Kiedroń, Stanisław/Kowalska-Szubert, Agata. (eds.) (2004): Thesaurus polyglottus et flores quadrilingues. Festschrift für Stanisław Prędota zum 60. Geburtstag. Wrocław, Oficyna Wydawnicza ATUT - Wrocławskie Wydawnictwo Oświatowe: 247-262.

Plattform Codecheck www.codecheck.info/suesswaren_snacks/frucht_weingummi/ean_76 40104959014/id_819949/Biolini_Salbei_Bonbons.pro [25.04.13].

Röhrich, Lutz (1994): Lexikon der Sprichwörtlichen Redensarten. Freiburg etc.: Herder/Spektrum.

Röhrich, Lutz/Mieder, Wolfgang (1977): Das Sprichwort. Stuttgart: J.B. Metzlersche Verlagsbuchhandlung/Carl Ernst Poeschel.

Sandig, Barbara (1994): Europhras 92. Tendenzen der Phraseologieforschung. Bochum, Brockmeyer.

Seiler, Friedrich (1922): Deutsche Sprichwörterkunde. München: Beck.

Wander, Karl Friedrich Wilhelm (2008): Deutsches Sprichwörter-Lexikon. Berlin: Directmedia Publishing. www.zeno.org/Wander-1867 [05.07.2015]. (= Digitale Bibliothek 034). 